\title{
Prospective study of primary amenorrhoea and its management in a rural tertiary centre
}

\author{
Pavanaganga A.*, Sai Prathyusha Ivalla, Nagarathnamma R., Sailakshmi M. P. A.
}

Department of Obstetrics and Gynecology, Rajarajeswari Medical College and Hospital, Bangalore, Karnataka, India

Received: 23 July 2019

Accepted: 12 August 2019

*Correspondence:

Dr. Pavanaganga A.,

E-mail: pavanagangaa@gmail.com

Copyright: (c) the author(s), publisher and licensee Medip Academy. This is an open-access article distributed under the terms of the Creative Commons Attribution Non-Commercial License, which permits unrestricted non-commercial use, distribution, and reproduction in any medium, provided the original work is properly cited.

\begin{abstract}
Background: Amenorrhoea (absence of menstruation) is a symptom of varied causes. It results from dysfunction of hypothalamic-pituitary ovarian axis, uterus and vagina. It is a major concern for pubertal girls and their family members. It has a major impact on the physical, mental, psychological and social life of the girl and her family. The objective of the present study was to evaluate the aetiology and management of primary amenorrhoea in young adolescent girls.

Methods: It was a prospective study conducted for a period of 2 years from August 2016 to July 2018 at Rajarajeswari medical college and hospital. Patients presenting with history of amenorrhoea that is, absence of menses by the age of 13 years with no visible development of secondary sexual characteristics or by 15 years of age with the presence of normal secondary sexual characteristics were included in our study. Cases of secondary amenorrhoea were excluded. Detailed history, examination, investigations and management was documented and analysed.

Results: A total of 25 patients of primary amenorrhea were studied during the study period. In our study outflow tract anomalies were the commonest cause of amenorrhoea accounting for $84 \%$, of which imperforate hymen (32\%) and Mayer Rokitansky Küster Hauser syndrome (MRKH) 36\% were the two most common Mullerian anomaly causing primary amenorrhoea. Gonadal dysgenesis accounted for $12 \%$ of the cases. Amenorrhoea was the commonest complaint patients presented with accounting to $76 \%$, followed by cyclical pain abdomen accounting for $16 \%$ of cases.

Conclusions: Primary amenorrhoea is multifactorial and is of major concern among adolescent girls. Early diagnosis and intervention has an impact on the physical and psychological wellbeing of the girl.
\end{abstract}

Keywords: Gonadal dysgenesis, Mayer Rokitansky Küster Hauser syndrome, Primary amenorrhoea, Vaginoplasty

\section{INTRODUCTION}

Amenorrhoea is defined as absence of menstruation. It can be a transient, intermittent, or permanent condition resulting from dysfunction of the hypothalamus, pituitary, ovaries, uterus, or vagina. It is classified as primary and secondary amenorrhoea.

Primary amenorrhoea is defined as absence of menstruation by 14 years of age without secondary sexual development or absence of menstruation by 16 years with secondary sexual development. ${ }^{1}$ Secondary amenorrhoea is defined as cessation of previously regular menses for 3 months or cessation of previously irregular menses for 6 months. $^{2}$

According WHO incidence of amenorrhoea is $2-5 \%$ and is the sixth largest cause for female infertility. ${ }^{3}$ Incidence of primary amenorrhoea is $1-2 \% .^{3,4}$ Although incidence of primary amenorrhoea is small, it needs timely evaluation and intervention as it has an impact on the physical and psychological wellbeing of the girl. 
Menarche is attainment of first menstruation, it heralds attainment of womanhood. It empowers the continuum of life and procreation. Attainment of menarche is celebrated with grandeur in few communities. ${ }^{5}$ Menarche marks the end of a series of events which begin in cerebral cortex and hypothalamus and terminates in uterine and ovarian tissues. It includes events such as thelarche, pubarche, growth spurt and ultimately culminating in menarche. Normal menstruation is due to co-ordinated activity of Hypothalamus-pituitary-ovarian axis. Any interruption in the normal functioning of hypothalamuspituitary ovarian axis results in amenorrhoea. ${ }^{6}$

The causes of primary amenorrhea are classified into five groups based on the organs involved in the aetiology. ${ }^{6}$

- Compartment 1: End-organ failure or out-flow tract obstruction

- Compartment 2: Gonadal failure

- Compartment 3: Pituitary cause

- Compartment 4: Hypothalamic cause

- Other causes.

Though the incidence of primary amenorrhoea is less it has an enormous impact on psychological and social health of adolescent girls, hence this study was conducted in our tertiary rural centre to evaluate the aetiology and management of primary amenorrhoea in young adolescent girls. Early diagnosis and timely intervention prevent complications.

\section{METHODS}

It was a prospective study conducted for a period of 2years from August 2016 to July 2018 at Rajarajeswari Medical College and Hospital. Patients presenting to outpatient department with history of primary amenorrhoea that is, absence of menses by the age of 14 years with no visible development of secondary sexual characteristics or by 16 years of age with the presence of normal secondary sexual characteristics were included in our study. Cases of secondary amenorrhoea was excluded.

\section{Definition of primary amenorrhoea}

Absence of menstruation by 14 years of age without secondary sexual development or absence of menstruation by 16 years with secondary sexual development.

\section{Definition of secondary amenorrhoea}

Cessation of previously regular menses for 3 months or cessation of previously irregular menses for 6 months.

After taking informed consent, a brief history was taken which included age at presentation, socioeconomic status, eating and exercise patterns, cyclic abdominal pain, any disorder of smell perception, maternal age at menarche, presence of galactorrhoea, history of tuberculosis, family history of primary or secondary amenorrhea, mental retardation, history of any other significant illness was also elicited.

Physical examination included examination of height, weight, built, BMI (body mass index), presence of secondary sexual characters, thyroid palpation, Tanner staging of breast and pubic hairs, local examination of external genitalia, Rectal examination was done to assess anatomic or mullerian anomalies. Dysmorphic features such as a webbed neck or low hairline and other features of Turner syndrome were recorded.

Laboratory workup included serum luteinizing hormone, follicle-stimulating hormone, prolactin, and thyroid function tests, serum free and total testosterone, dehydroepiandroster-one sulphate, oestradiol and 17 Hydroxy Progesterone. A complete blood count and comprehensive metabolic panel, Mantoux test and X-ray of chest was done if history or examination was suggestive of chronic disease. Diagnostic hysteroscopy and endometrial aspirate were taken for histopathology and for TB-PCR (Tubercular Bacillus-polymerase chain reaction) in suspected cases. Pregnancy test was done wherever necessary.

Pelvic ultrasonography (transabdominal or transvaginal) was done for presence of uterus, adnexa, and any reproductive tract anomaly. In cases where USG was inconclusive, MRI was done to visualize Mullerian structures, gonads and renal anomalies. Laparoscopy and hysteroscopy were also done for diagnosis and management as and when indicated. Cytogenetic study for karyotype detection was done in cases of hypergonadotropic hypogonadism and those with symptoms of androgen excess.

\section{RESULTS}

Analysis of 25 cases of primary amenorrhoea was done during our 2-year study period. Patients presenting to Outpatient department with complaints of amenorrhoea were subjected to detailed analysis which included history taking, examination, appropriate laboratory investigations and imaging studies.

Table 1: Age of presentation.

\begin{tabular}{|lll|}
\hline Age (Years) & No. cases & Percentage \\
\hline $16-20$ & 22 & $88 \%$ \\
\hline $20-25$ & 1 & $4 \%$ \\
\hline$>25$ & 2 & $8 \%$ \\
\hline
\end{tabular}

Most patients in our study belonged to the age group between $16-20$ years $(88 \%)$. A total of $n=22$ patients belonged to this age group as shown in Table 1. Two patients $(8 \%)$ were more than 25 years of age. Nineteen patients belonged to low socioeconomic status, four were 
from lower middle class and two belonged to upper middle class.

Table 2: Presenting complaints.

\begin{tabular}{|lll|}
\hline Complaints & No. of cases & Percentage \\
\hline Amenorrhoea & 19 & $76 \%$ \\
\hline Cyclical pain abdomen & 4 & $16 \%$ \\
\hline Abdominal swelling & 1 & $4 \%$ \\
\hline Infertility & 1 & $4 \%$ \\
\hline
\end{tabular}

Amenorrhoea was the commonest complaint patients presented with $(n=19) 76 \%$, followed by cyclical pain abdomen $(n=4) 16 \%$ of cases as shown in Table 2 . Abdominal swelling was noted in one case, swelling was due the gonads in the deep inguinal ring, that case was diagnosed as androgen insensitivity syndrome. Two patients presented with history of infertility; on detailed analysis she was diagnosed to have primary amenorrhoea etiological cause was genital tuberculosis.

Table 3: Causes of primary amenorrhoea.

\begin{tabular}{|llll|}
\hline $\begin{array}{l}\text { Sr. } \\
\text { no. }\end{array}$ & Causes & $\begin{array}{l}\text { No. of } \\
\text { cases }\end{array}$ & Percentage \\
\hline 1 & $\begin{array}{l}\text { Mayer Rokitansky } \\
\text { Küster Hauser }\end{array}$ & 9 & $36 \%$ \\
\hline 2 & Imperforate hymen & 8 & $32 \%$ \\
\hline 3 & Gonadal dysgenesis & 3 & $12 \%$ \\
\hline 4 & $\begin{array}{l}\text { Transverse vaginal } \\
\text { septum }\end{array}$ & 1 & $4 \%$ \\
\hline 5 & $\begin{array}{l}\text { Androgen insensitivity } \\
\text { syndrome }\end{array}$ & 1 & $4 \%$ \\
\hline 6 & Tuberculosis & 2 & $8 \%$ \\
\hline 7 & Physiological delay & 1 & $4 \%$ \\
\hline
\end{tabular}

In our study outflow tract anomalies were the commonest cause of amenorrhoea accounting for $84 \%$, of which imperforate hymen (32\%) and Mayer Rokitansky Küster Hauser syndrome (36\%) were the two most common Mullerian causes of primary amenorrhoea as shown in Table 3. Gonadal dysgenesis accounted for $12 \%$ of the cases followed by genital tuberculosis $8 \%$, transverse septum $4 \%$ and androgen insensitivity syndrome $4 \%$.

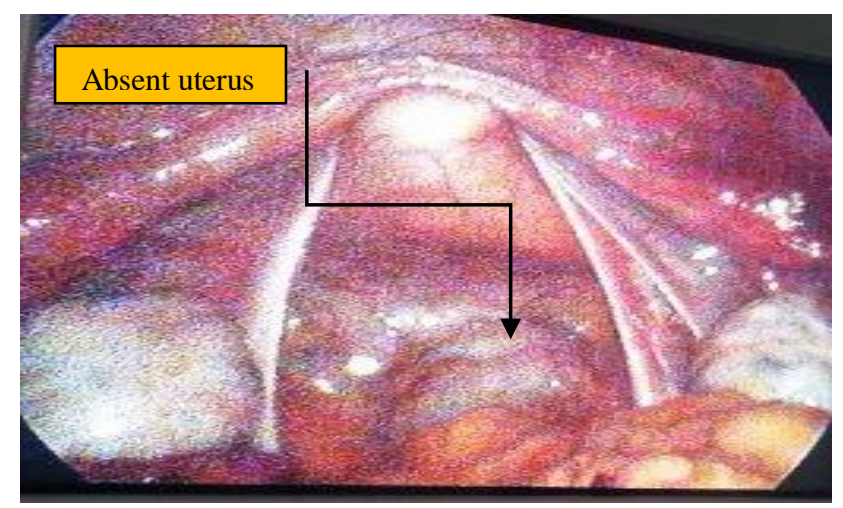

Figure 1: MRKH syndrome.

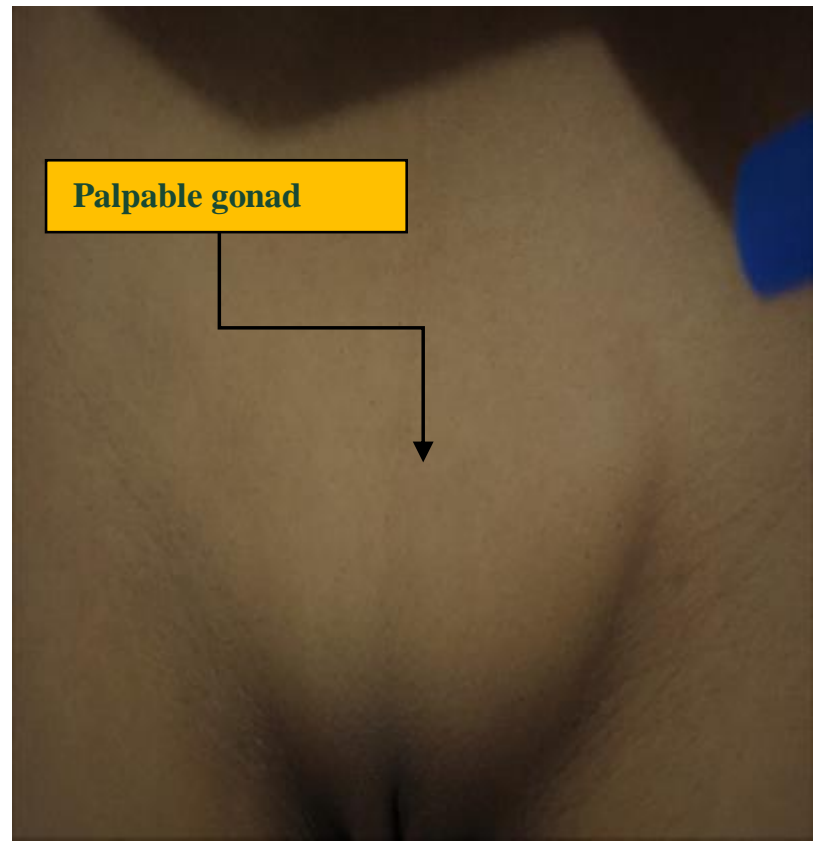

Figure 2: Androgen insensitivity syndrome showing palpable gonads in inguinal region.

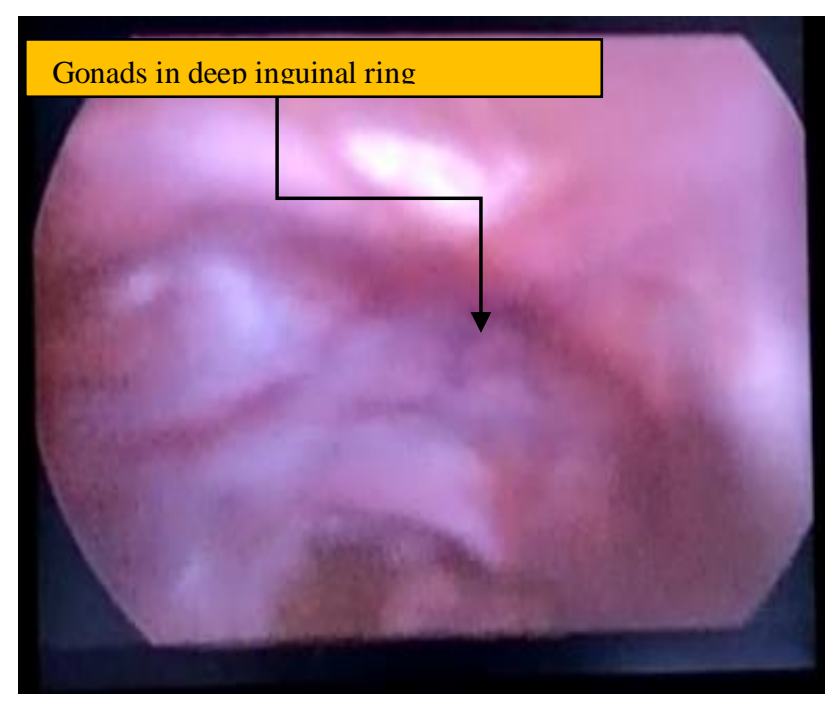

Figure 3: Laparoscopic view of absent uterus in androgen insensitivity syndrome.

\section{DISCUSSION}

Primary amenorrhoea is a major concern for pubertal girls and their parents especially regarding the reproductive life. Primary amenorrhoea is multi-factorial, causes have been compartmentalized. Girls presenting with amenorrhoea must be evaluated meticulously. Detailed history and physical examination including evidence of psychogenic dysfunction or emotional stress, family history of apparent genetic anomalies with a focus on nutritional status, abnormal growth and development, the presence of a normal reproductive tract and examination is followed by step by step investigations, which includes hormonal assay and pelvic imaging. 
Cytogenetic studies are done in cases with hypergonadotropic hypogonadism and girls with androgenic features.

In this study most patients presented at the age group of 16-20 years (88\%), similar results were obtained in two studies conducted by Anita et al, at Vani Vilas Government hospital at Karnataka and Civil hospital Ahmedabad.5,6 Nineteen patients belonged to low socioeco-nomic status, four were from lower middle class and two belonged to upper middle class.

Presenting complaint was amenorrhoea in $76 \%(n=19)$ of patients in our study, similar results were noted in studies done by Kriplani A et al, All India Medical Sciences New Delhi, Anitha et al Vani Vilas Government hospital at Karnataka and Civil hospital Ahmedabad were similar. 5,6 Cyclical abdominal pain was noted in $16 \%$ of cases in our study, similar results was noted in study done by Anitha et al, at Vani Vilas Government hospital at Karnataka.

In our study the commonest cause for amenorrhoea was Mullerian anomaly $n=20(84 \%)$, of these eight patients had imperforate hymen $(32 \%)$, nine had Mayer Rokitansky Küster Hauser syndrome (36\%), one patient had transverse septum (4\%) and two had genital tuberculosis (8\%). Mullerian anomaly was the commonest cause of amenorrhoea in studies done by Kriplani A et al (47\%), Tanmahasamut $\mathrm{P}$ et al. ${ }^{1}$ (39.7\%), Anita et al, Vani Vilas Government hospital at Karnataka (32\%) and Tushar et al, Civil hospital Ahmedabad $(44 \%){ }^{5-9}$ Studies done in India and other Asian countries have reported higher incidence of Mullerian anomalies as the cause for primary amenorrhoea in contrast to western studies where gonadal dysgenesis is the commonest cause.

In our study Nine patients were diagnosed with Mayer Rokitansky Küster Hauser syndrome. Diagnostic laparoscopy was done for all cases, six patients had absent uterus, both ovaries were normal, and vagina was absent, Figure 1 shows laparoscopic findings. Three patients had hypoplastic uterus. Two patients had associated renal anomalies. Out of these two patients, one had absent left kidney other one patient had ectopic kidney. The patient with absent left kidney also had congenital heart disease with pulmonary valve stenosis. Four patients with Mayer Rokitansky Küster Hauser syndrome underwent Mc Indoe's vaginoplasty. Three patients were lost for follow up; one patient refused surgical intervention. All these patients were counseled regarding their reproductive life and future fertility options like surrogacy for having biological child.

One patient (4\%) had transverse vaginal septum, she underwent vaginal septal resection. Study done by Tushar et al, done at civil hospital Ahmedabad showed three patients out of $25(12 \%)$ had transverse vaginal septum. ${ }^{6}$ Study of Kriplani A et al, had five cases of transverse vaginal septum. Diagnostic laparoscopy done for our patient showed well developed right horn with hematometra and a left rudimentary horn attached to inguinal ligament. She had septal resection and was put on combined oestrogen progesterone pills for three cycles. Patient is having regular menstrual cycles.

Three patients in our study had gonadal dysgenesis $n=3$ $(12 \%)$. All three patients underwent cytogenetic studies. Karyotyping showed two patients had 46XX gonadal dysgenesis, one patient had 45XO Turner Syndrome. Studies of Akthar A et al, Kriplani A et al, Tahir et al and Shanoli et al showed an incidence of $13.3 \%, 20.5 \%$ and $23.9 \%, 35.8 \%$, respectively and Studies done by Anita et al at Vani Vilas Government hospital showed an incidence of $32 \% .^{1,10-12}$ Patients with primary gonadal failure were put on combined hormone therapy with oestrogen for 21 days (conjugated equine oestrogen 0.625 $\mathrm{mg}$ daily) and progesterone (medroxyprogesterone acetate $10 \mathrm{mg}$ daily) in last 12 days of the month. They were also given calcium and vitamin D supplementation to prevent bone loss. All three patients were counseled regarding their reproductive life.

One patient had androgen insensitivity syndrome. She had palpable gonads as shown in Figure 2 and 3. Karyotyping showed 46XY chromosomes. She underwent bilateral gonadectomy. Patient counseled regarding sexual health and reproductive life.

Tuberculosis was diagnosed in two patients. Patient underwent diagnostic laparoscopy which revealed - retort shaped $\mathrm{B} / \mathrm{L}$ fallopian tubes in one patient and left tuboovarian complex and right hydro salpinx in another case. Tubercles present over surface of uterus, ovaries and omentum. Intra peritoneal flimsy omental adhesions noted. Biopsy of tubercle was taken. Mantoux test, Sputum AFB culture was negative, Real time PCR (sample from blood and omental tubercle biopsy) was Positive for Mycobacterium Tuberculosis complex. Patients were started on ATT as per RNTCP and were followed up. Female genital tuberculosis is prevalent in India and it is one of the important causes of primary amenorrhea with guarded prognosis. ${ }^{13,14}$ It affects all the reproductive organs including uterus, fallopian tubes, and ovaries leading to amenorrhea either primary or secondary amenorrhea. ${ }^{13}$ It has worst outcome when endometrial lining is affected, and it leads to endometrial damage thereby causing primary amenorrhea.

\section{CONCLUSION}

Primary amenorrhoea is multi-factorial and enigmatic. It is a major concern for pubertal girls and their family members. It has a major impact on the physical, mental, psychological and social life of the girl and family. Though there is a social and psychological stigma related to primary amenorrhoea, parents need to seek early medical help for early diagnosis and intervention. Multidisciplinary approach involving gynaecologist, geneticist, psychologist and paediatrician should be 
followed for individualizing the management and counselling. Patient and the family should be counselled about the future sexual health, fertility and hormone replacement. Treatment and prognosis in terms of future fertility depends on the primary aetiology of amenorrhea.

Funding: No funding sources

Conflict of interest: None declared

Ethical approval: The study was approved by the Institutional Ethics Committee

\section{REFERENCES}

1. Kriplani A, Goyal M, Kachhawa G, Mahey R, Kulshrestha V. Aetiology and management of primary amenorrhoea: a study of 102 cases at tertiary centre. Taiwan J Obstet Gynaecol. 2017;56(6):761-4.

2. Kochar Kaur K, Allahbadia GN, Singh M. An update on the causes of primary and secondary amenorrhea along with aetiopathogenesis and therapeutic management. Avid Sci Monog Series. 2016:1-43.

3. Dutta UR, Ponnala R, Pidugu VK, Dalal AB. Chromosomal abnormalities in amenorrhea: a retrospective study and review of 637 patients in south India. Arch Iran Med. 2013;16(5):267e70.

4. Balampa P, Rabindran, Pavani. Primary amenorrhoea - a single centre experience of 38 cases. Obg Rev J Obstet Gynecol. 2015;1(1):9-13.

5. Anitha GS, Tejaswini KK, Murthy GS. Clinical study of primary amenorrhoea. J South Asian Federation Obstet Gynaecol. 2015;7(3):158-66.

6. Shah TM, Lalcheta FR. Case series of primary amenorrhoea. Int J Curr Res. 2018;10(6):70666-9.

7. Tanmahasamut $\mathrm{P}$, Rattanachaiyanont M, Dangrat C, Indhavivadhana S, Angsuwattana S, Techatraisak K.
Causes of primary amenorrhea: a report of 295 cases in Thailand. J Obstetric Gynaecology Res. 2012;38:297e301.

8. Parikh RM. Mullerian anomalies: a cause of primary amenorrhea. Int J Reprod Contracept Obstet Gynecol. 2013;2(3):393-7.

9. Andallu R. India study of incidence, causes and outcome of management of primary amenorrhoea. Indian Obstet Gynaecol. 2016;6:2.

10. Akhtar A, Sudhir PS. Etiological evaluation of primary amenorrhea in a rural teaching hospital. JBCR 2018;5(1):3-20.

11. Ghosh S, Roy S, Pal P, Dutta A, Halder A. Cytogenetic analysis of patients with primary amenorrhea in Eastern India. J Obstet Gynaecol. 2018;38(2):1.

12. Malla TM, Dar FA, Pandith AA, Zargar MH. Frequency and pattern of cytogenetic alterations in primary amenorrhea cases of Kashmir, North India. Egypt J Med Human Genet. 2016;17(1):25-31.

13. Singh N, Sumana G, Mittal S. Genital tuberculosis: a leading cause for infertility in women seeking assisted conception in North India. Arch Gynecol Obstetric. 2008;278(4):325e7.

14. Tripathy SN, Tripathy SN. Infertility and pregnancy outcome in female genital tuberculosis. Int J Gynecol Obstetric. 2002;76:159e63.

Cite this article as: Pavanaganga A, Ivalla SP, Nagarathnamma R, Sailakshmi MPA. Prospective study of primary amenorrhoea and its management in a rural tertiary centre. Int J Reprod Contracept Obstet Gynecol 2019;8:3442-6. 